\title{
Mex3a expression and survival analysis of bladder urothelial carcinoma
}

\author{
Jing-wen Shi ${ }^{1}$ and Ying Huang ${ }^{1}$ \\ ${ }^{1}$ Department of Ultrasound, Shengjing Hospital of China Medical University, 110004, Shenyang, China \\ Correspondence to: Ying Huang, email: huangying712@163.com \\ Keywords: mex3a, bladder urothelial carcinoma, overall survival, TCGA, pathology
}

Abbreviations: BLCA: Bladder Urothelial Carcinoma; TCGA: The Cancer Genome Atlas; OS: overall survival; AJCC: American Joint Committee on Cancer; CIS: carcinoma in situ

Received: April 18, $2017 \quad$ Accepted: May 27, $2017 \quad$ Published: June 07, 2017

Copyright: Shi et al. This is an open-access article distributed under the terms of the Creative Commons Attribution License 3.0 (CC BY 3.0), which permits unrestricted use, distribution, and reproduction in any medium, provided the original author and source are credited.

\section{ABSTRACT}

Objective: Bladder urothelial carcinoma is a common tumor in humans and a multifactorial disease. The gene mex3a is associated with tumor formation and may promote cell proliferation and migration. Therefore, this study aimed to determine the relationship between mex3a and bladder urothelial carcinoma.

Methods: The clinical and RNA sequencing expression data in patients with bladder urothelial carcinoma were downloaded from the The Cancer Genome Atlas data portal. A total of $\mathbf{4 1 2}$ bladder urothelial carcinoma samples were available in the database, for which the clinical information was acquired, of which 412 are RNA sequencing samples with a total of 19 paired samples. Univariate and multivariate Cox analyses and univariate logistic regression analysis were conducted using the software SPSS version $\mathbf{2 2 . 0}$ and $\boldsymbol{P}<\mathbf{0 . 0 5}$ was considered statistically significant.

Results: The results of the independent $t$-test of 19 paired samples indicated that the expression level of mex3a was significantly higher in tumor tissues compared with adjacent normal tissues. Mex3a expression as a categorical dependent variable was not associated with overall survival, and the overall survival of bladder urothelial carcinoma was associated with the group of age, cancer status, lymphatic vascular invasion, pathological stage, pathological size, and pathological lymph metastasis. The multivariable Cox model adjusted for the group of mex3a expression level, age, gender, tumor status, and pathological stage showed that only the age and cancer status groups were associated with the overall survival.

Conclusion: Mex3a expression was not a poor prognostic factor of bladder urothelial carcinoma. Moreover, the expression levels of mex3a in the papillary type of bladder urothelial carcinoma were higher than those of the non-papillary type.

\section{INTRODUCTION}

Bladder urothelial carcinoma (BLCA) is one of the most common tumors in humans and the second most frequently diagnosed genitourinary tumor $[1,2]$. It has been reported that the occurrence and development of BLCA are multi-factorial, multi-stage, and involves multigene changes [3-5]. Although recent methods that employ novel technologies may be recognized as a promising option for bladder carcinoma treatment in the near future
$[6,7]$, surgical operation is the primary method for treating bladder carcinoma, and chemotherapy is thought to be an effective adjunctive therapy to avoid the recurrence and metastasis of BLCA [8]. Considering that immunotherapy has become a treatment paradigm in bladder cancer and that drug resistance has increased, novel biomarkers are necessary and helpful to improve risk stratification and optimize the therapeutic choice of BLCA [9-11].

The gene mex $3 a$ is associated with tumor formation and may promote cell proliferation and tumor metastasis 
[12]. In this study, we did a thorough search for novel fusion transcripts in bladder cancer using RNA sequencing and sought to determine the effect of mex $3 a$ expression on the overall survival of BLCA. We analyzed the mex $3 a$ mRNA expression level in cases of BLCA and evaluated its prognostic value and whether mex $3 a$ could be a biomarker and a potential therapeutic target in high-risk BLCA.

\section{RESULTS}

\section{Patient characteristics}

Both clinical and gene expression data of the 412 primary tumors were acquired from the TCGA bladder cancer database, with data from 19 paired samples. Patients' characteristics are shown in Table 1. Seventy-four percent of the cohort patients were men, and 26.2\% were women. Approximately 79.4\% were Caucasian, 5.6\% were black of African-American descent, and 10.7\% were Asian. Disease stages II, III, and IV corresponded to $32.3 \%, 34.2 \%$, and $33.0 \%$ of the population, respectively. More than $50 \%$ of tumors $(58 \%$, $\mathrm{n}=239$ ) were of pathological $\mathrm{n} 0$, and $11.4 \%, 18.4 \%$, and $1.9 \%$ of the tumors corresponded to pathologic stage $\mathrm{n} 1, \mathrm{n} 2$, and $\mathrm{n} 3$, respectively. In addition, $30.1 \%, 47.6 \%$, $14.3 \%$ of the sample presented pathologic T2, T3, and T4 tumors, respectively, according to the American Joint Committee on Cancer (AJCC). Most tumor subtypes $(66.5 \%, n=274)$ were non-papillary, and papillary tumors were detected in $32.3 \%$ of the sample $(n=133)$. The outcomes of primary therapy were growing tumors $(10.2 \%)$, complete remission $(37.4 \%)$, solid tumors $(5.6 \%)$, and partial remission (4.1\%). Ninety-four percent $(n=388)$ of the tumors were high-grade whereas $5.1 \%$ $(\mathrm{n}=21)$ were low-grade. More than 50\% $(57.0 \%, \mathrm{n}=235)$ of the patients were tumor-free whereas $33.3 \%(n=137)$ had tumors. Thirty-two percent $(n=132)$ of the patients did no present lymphatic vascular invasion whereas $37.1 \%(n=153)$ had a vascular invasion. The median

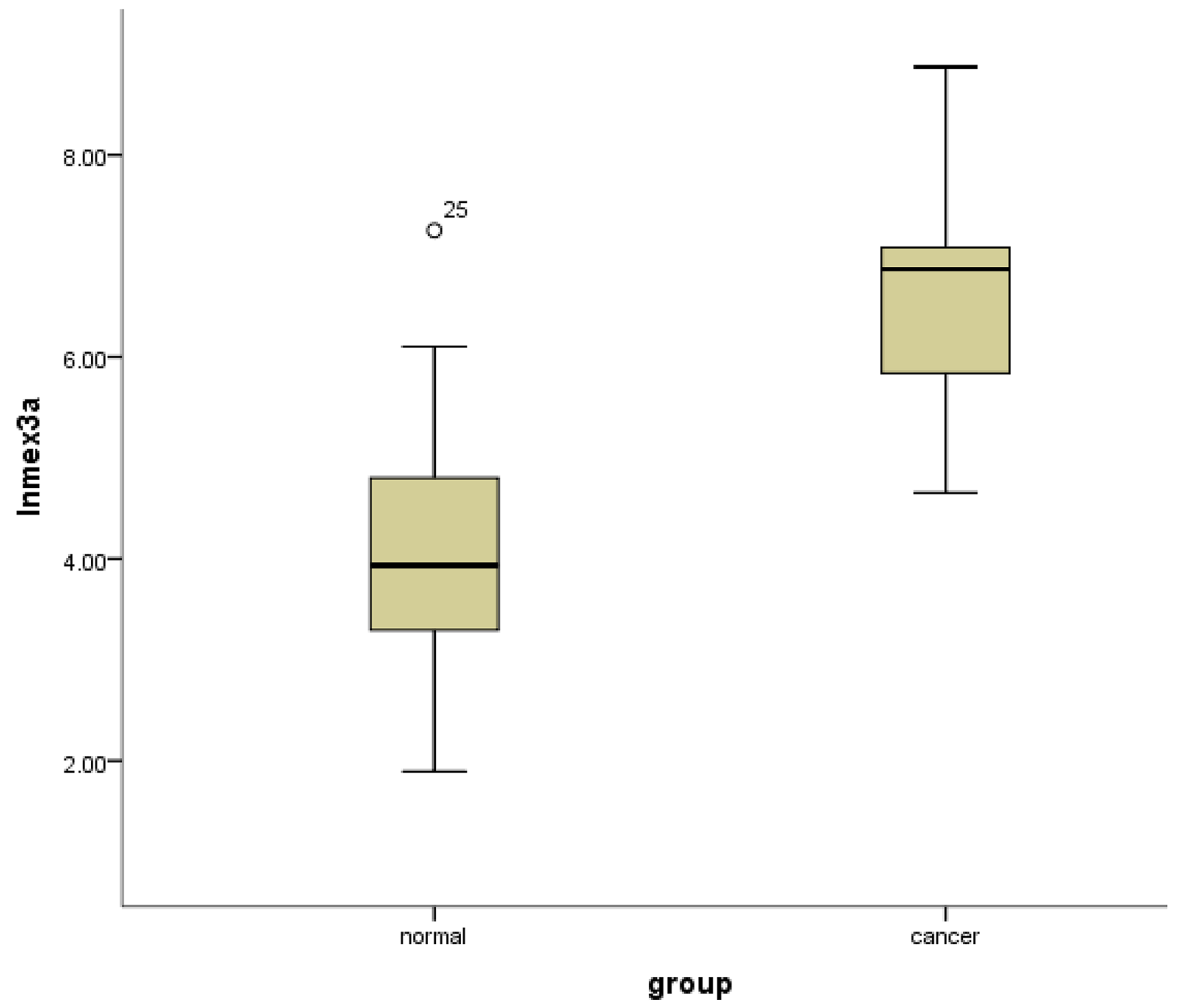

Figure 1: Box-plots shows the $\ln (\mathrm{Mex3a})$ value between cancer tissues and normal adjacent tissues. 
Table 1: Characteristics of BLCA patients

\begin{tabular}{|c|c|c|}
\hline Characteristic & Total & $\%$ \\
\hline Gender & 304 & 73.8 \\
\hline male & 108 & 26.2 \\
\hline \multicolumn{3}{|l|}{ female } \\
\hline \multicolumn{3}{|l|}{ Vital state } \\
\hline alive & 303 & 73.5 \\
\hline dead & 109 & 26.5 \\
\hline \multicolumn{3}{|l|}{ Pathologic $n$} \\
\hline N0 & 239 & 58.0 \\
\hline N1 & 47 & 11.4 \\
\hline $\mathrm{N} 2$ & 76 & 18.4 \\
\hline N3 & 8 & 1.9 \\
\hline \multicolumn{3}{|l|}{ Pathologic t } \\
\hline $\mathrm{T} 2$ & 124 & 30.1 \\
\hline $\mathrm{T} 3$ & 196 & 47.6 \\
\hline $\mathrm{T} 4$ & 59 & 14.3 \\
\hline \multicolumn{3}{|l|}{ Pathologic stage } \\
\hline stage ii & 133 & 32.3 \\
\hline stage iii & 141 & 34.3 \\
\hline stage iv & 136 & 33.0 \\
\hline \multicolumn{3}{|l|}{ Cancer status } \\
\hline with tumor & 137 & 33.3 \\
\hline tumor free & 235 & 57.0 \\
\hline \multicolumn{3}{|l|}{ Subtype } \\
\hline non-papillary & 274 & 66.5 \\
\hline papillary & 133 & 32.3 \\
\hline \multicolumn{3}{|c|}{ lymphatic vascular invasion } \\
\hline no & 132 & 32.0 \\
\hline yes & 153 & 37.1 \\
\hline
\end{tabular}

follow-up for the subjects alive at last contact was 588 days (range of 0-4684 days).

\section{Mex3a expression in tumor tissues and adjacent normal tissues}

It was demonstrated that the mean normalized expression of mex $3 a$ was $1191.153 \pm 1556.508$ in cancer and $167.466 \pm 323.095$ in adjacent normal tissues. The normalized fold change of mex $3 a$ mRNA in cancer and normal tissues ranged from 0.89 to 151.22 , and the mean fold change was $31.75 \pm 46.75$. The result of the independent sample $t$-test indicated that the level of mex $3 a$ expression was significantly higher in tumor tissues compared with adjacent normal tissues $(P=0.008)$ (Figure 1).

\section{Mex3a expression and association with clinical variables}

Mex $3 a$ expression data for the 412 BLCA patients were acquired from the TCGA database. The median expression of mex $3 a$ as a categorical dependent variable in the univariate analysis was not associated with OS $(P=0.673)$ (Figure 2). Other factors associated with OS 
are shown in Table 2 . The older group first diagnosed with BLCA had poor OS $(P=0.038$, hazard ratio $[\mathrm{HR}]=1.505)$ (Figure 3), and this result agrees with that of a previous study [13] and with the mortality due to bladder cancer in China [14]. BLCA patients with tumor had a 7.9-fold higher risk of death than tumor-free patients $(P<0.000$, $\mathrm{HR}=7.883$ ) (Figure 4). The increase in pathologic stage (Figure 5), pathological size, and pathological lymph metastasis (Figure 6) due to BLCA was associated with lower OS $(P<0.000)$. The lymphatic vascular invasion was also associated with lower OS $(P<0.000)$. The analysis of clinical-pathologic characteristics indicated that cancer subtype and gender were not associated with OS, ( $P=0.055$ and $P=0.513$, respectively). The result of logistic regression analysis indicated that high mex $3 a$ expression was associated with the papillary type of $\operatorname{BLCA}(P=0.006$, odds ratio $[\mathrm{OR}]=1.854$ ) (Figure 7$)$ and the older age group diagnosed with BLCA $(P=0.027, \mathrm{OR}=1.617)$ (Figure 8$)$, but was not associated with tumor status $(P=0.968)$ or pathologic stage $(P=0.816)$.

\section{Survival outcomes and multivariate analysis}

The multivariable Cox proportion hazards model adjusted for the mex $3 a$ expression group, and the group that included age, gender, cancer subtype, cancer status, pathologic stage, pathologic size, pathologic lymph metastasis, and lymphatic vascular invasion indicated that the older age group and the group with tumor were associated with $\mathrm{OS}(P=0.002, \mathrm{HR}=2.653, P<0.000$, $\mathrm{HR}=6.762$, respectively). However, there were no significant differences in mex $3 a$ and OS between these groups.

\section{DISCUSSION}

To date, a few studies have evaluated the effect of mex3a on tumor cells. Mex3a may be associated with tumors while has not yet seen its coverage on BLCA. A recent study from our research group indicated that mex $3 a$ could promote the proliferation of bladder urothelial cells

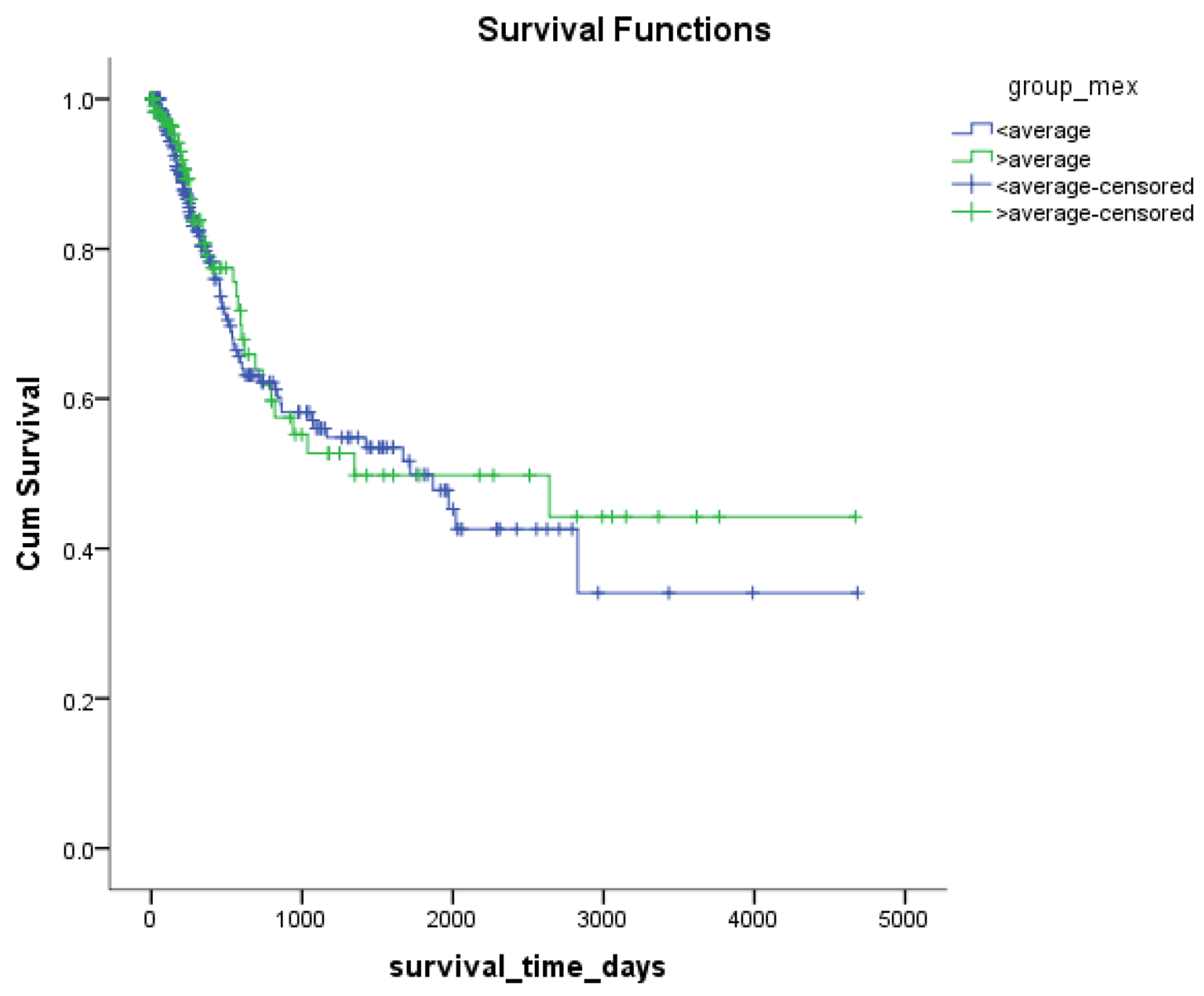

Figure 2: Kaplan-Meier plots of survival according to the high and low level of mex3a. 


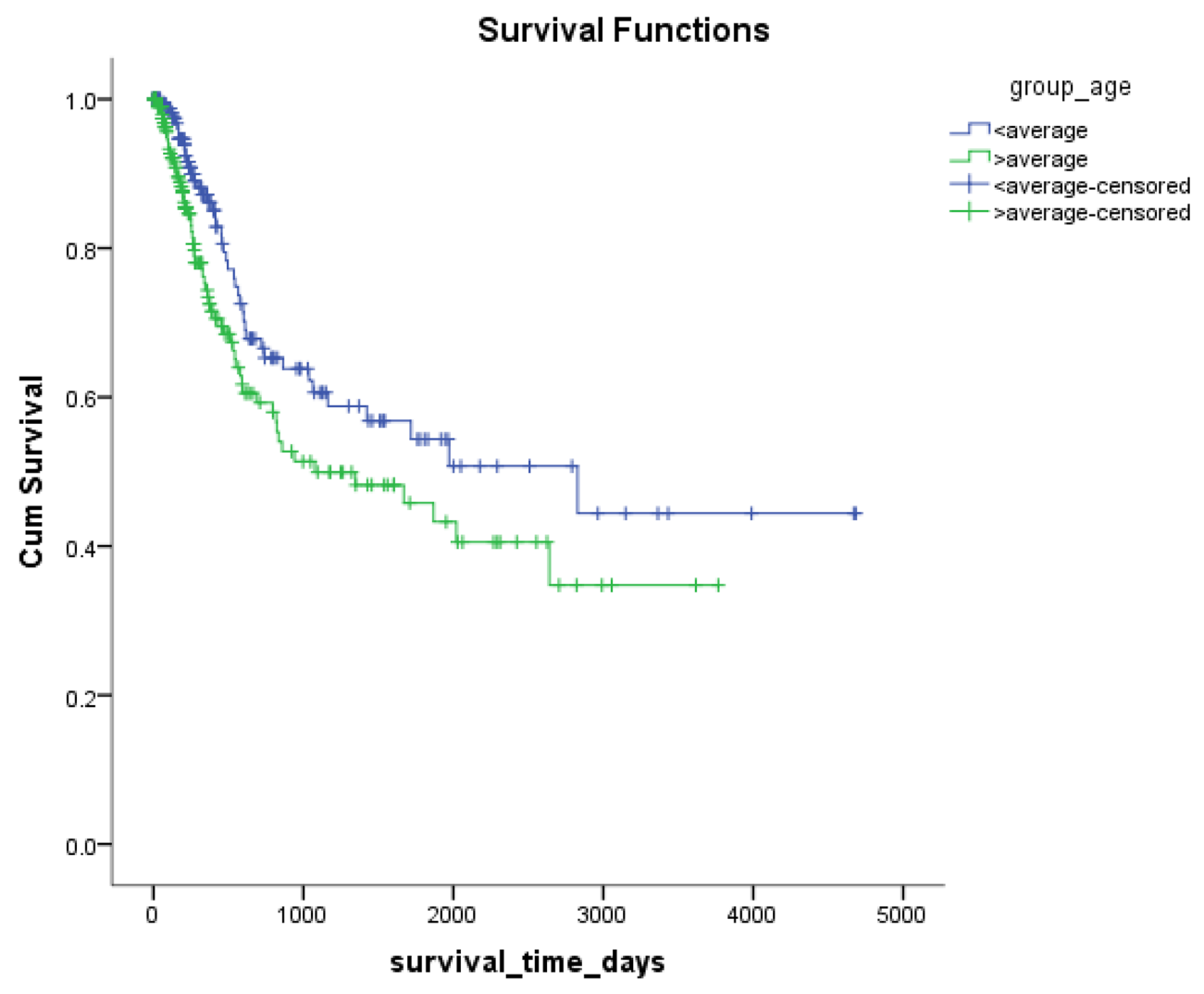

Figure 3: Kaplan-Meier survival plots according to the high and low age.

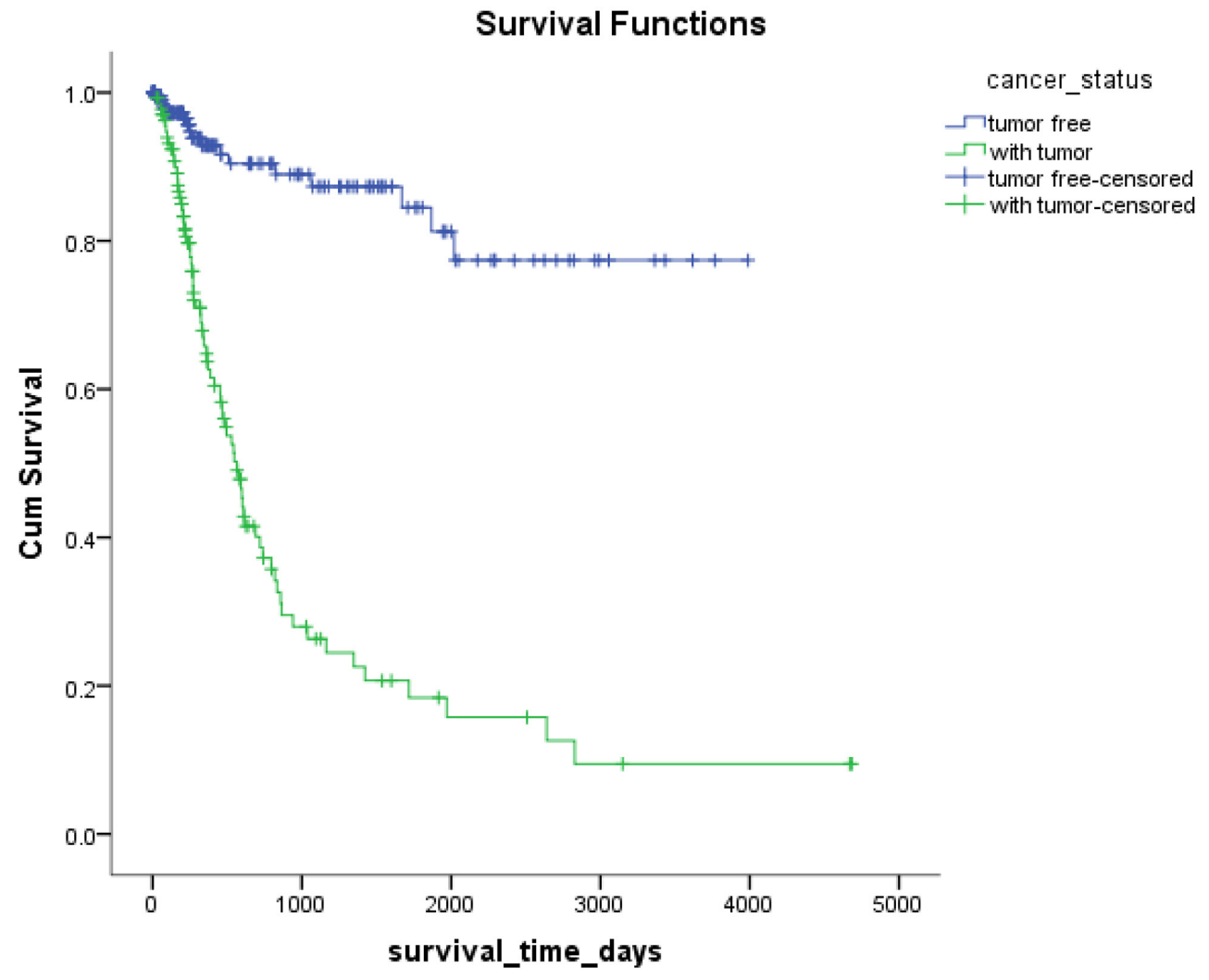

Figure 4: Kaplan-Meier survival plots according to the tumor status. 
Table 2: Cox regression univariate and multivariate analysis of OS for patients with BLCA

\begin{tabular}{|c|c|c|c|c|c|c|}
\hline & \multicolumn{3}{|c|}{ Univariate analysis } & \multicolumn{3}{|c|}{ Multivariate analysis } \\
\hline & HR & $95 \% \mathrm{CI}$ & P value & HR & $95 \% \mathrm{CI}$ & P value \\
\hline Group of age & 1.505 & $1.024 \sim 2.213$ & .038 & 2.653 & $1.430 \sim 4.919$ & .002 \\
\hline Group of Mex & .914 & $.604 \sim 1.385$ & .673 & .606 & $.303 \sim 1.210$ & .156 \\
\hline Gender & 1.147 & $.761 \sim 1.727$ & .513 & 1.495 & $.823 \sim 2.716$ & .186 \\
\hline subtype & .621 & $.381 \sim 1.010$ & .055 & .938 & $.459 \sim 1.915$ & .860 \\
\hline cancer status & 7.883 & $4.638 \sim 13.399$ & $<.000$ & 6.762 & $3.199 \sim 14.290$ & $<.000$ \\
\hline Pathologic grade & 1.938 & $1.495 \sim 2.513$ & $<.000$ & 1.045 & $.505 \sim 2.160$ & .906 \\
\hline Pathologic $t$ & 2.001 & $1.480 \sim 2.706$ & $<.000$ & 1.284 & $.795 \sim 2.074$ & .308 \\
\hline Pathologic $n$ & 1.631 & $1.319 \sim 2.017$ & $<.000$ & 1.069 & $.654 \sim 1.749$ & .790 \\
\hline $\begin{array}{l}\text { Lymphatic vascular } \\
\text { invasion }\end{array}$ & 2.897 & $1.748 \sim 4.800$ & $<.000$ & 1.639 & $.825 \sim 3.254$ & .158 \\
\hline
\end{tabular}

HR: hazard ratio; CI: Confidence interval.

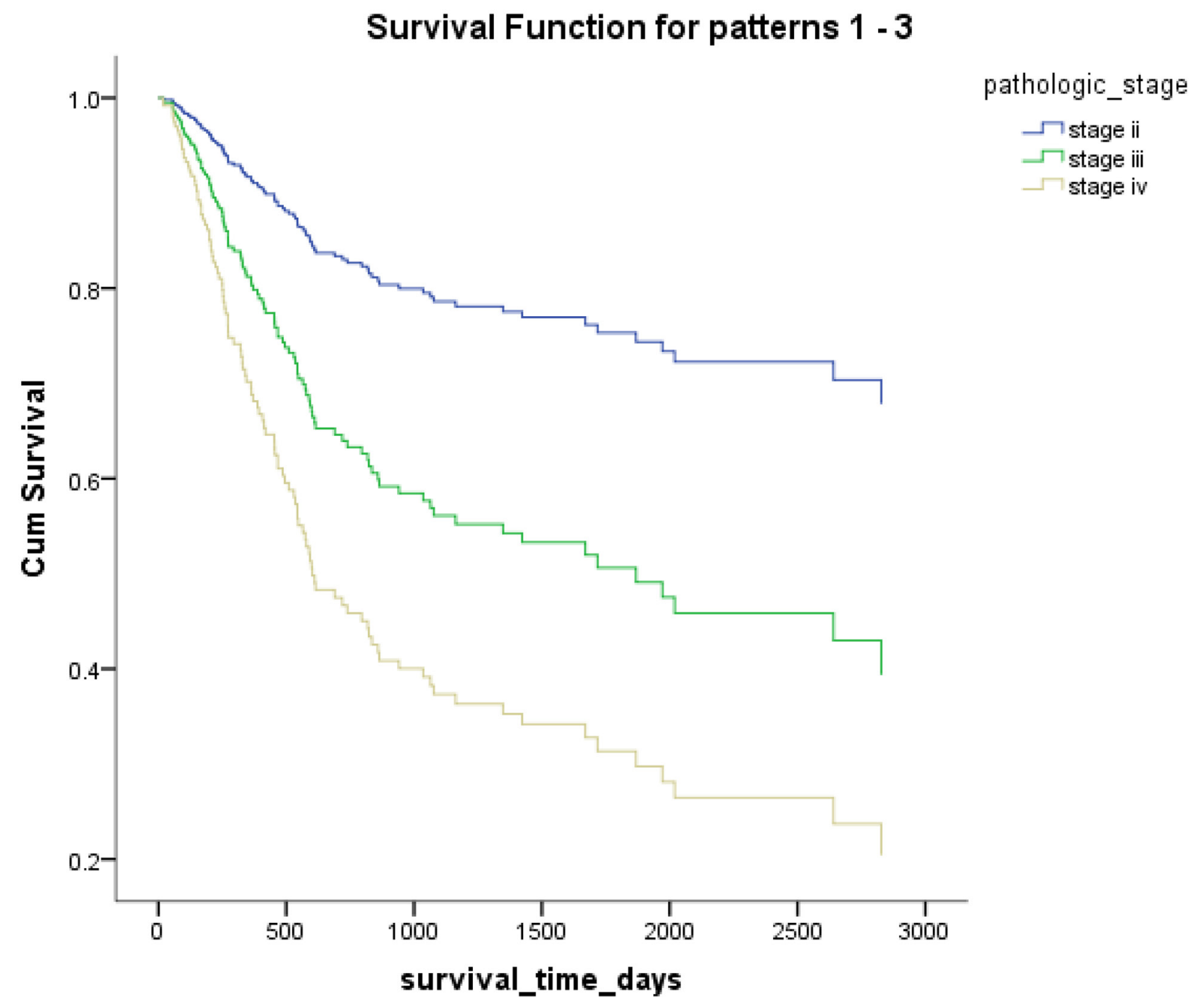

Figure 5: Overall survival according to the pathological stage. 
(in press). Therefore, herein we assessed the correlation between mex $3 a$ expression and OS in BLCA.

The mex-3 gene has a conserved region with $65-70$ amino acids. This region interacts with RNA and contains two K-homology domains and a family of four human genes homologous to Mex-3 (hMex-3), which was first characterized in heterogeneous nuclear ribonucleoproteins [15]. The protein Mex-3 was initially believed to regulate the localization and activity of several maternal factors with transducer activity and polarity cue in the Caenorhabditis elegans embryo [16]. Buchet-Poyau K. [17] identified four potential human genes homologous to the $C$. elegans mex-3 gene (hMex-3A, $-3 \mathrm{~B},-3 \mathrm{C}$, and $-3 \mathrm{D})$ located on distinct chromosomes at positions 1q22, $15 \mathrm{q} 25.2,18 \mathrm{q} 21.1$, and19p13.3, respectively.

Mex3a is located at complement $(156,072,013$ $156,081,998)$ and has 9986 base pairs. Jiang et al. [12] found that hMex-3D mRNA was ubiquitously expressed in all the cell lines and tissues tested by real-time polymerase chain reaction (RT-PCR) whereas the other three hMex-3 genes were expressed at varying levels in different tissues. It was reported that the inactivation of mex-3 by mutations could lead to embryonic death and defects in the anterior blastomere of the descendants. Jiang et al. [12] indicated that the knockout of gene $h M e x 3 a$ reduced the colonyforming ability of gastric cells in soft agar. Furthermore, transwell chamber and wound healing assays indicated that the knockout of $h M e x 3 a$ significantly affected the viability of cancer cells, and clinical correlation analysis indicated that the expression of $h M e x 3 a$ was significantly higher in cancer tissues than in normal tissues. Jiang et al. [12] demonstrated that $h M e x 3 a$ was involved in the regulation of tumorigenesis and that the aberrant activation of mex $3 a$ in human gastric cancer cells promoted cell proliferation

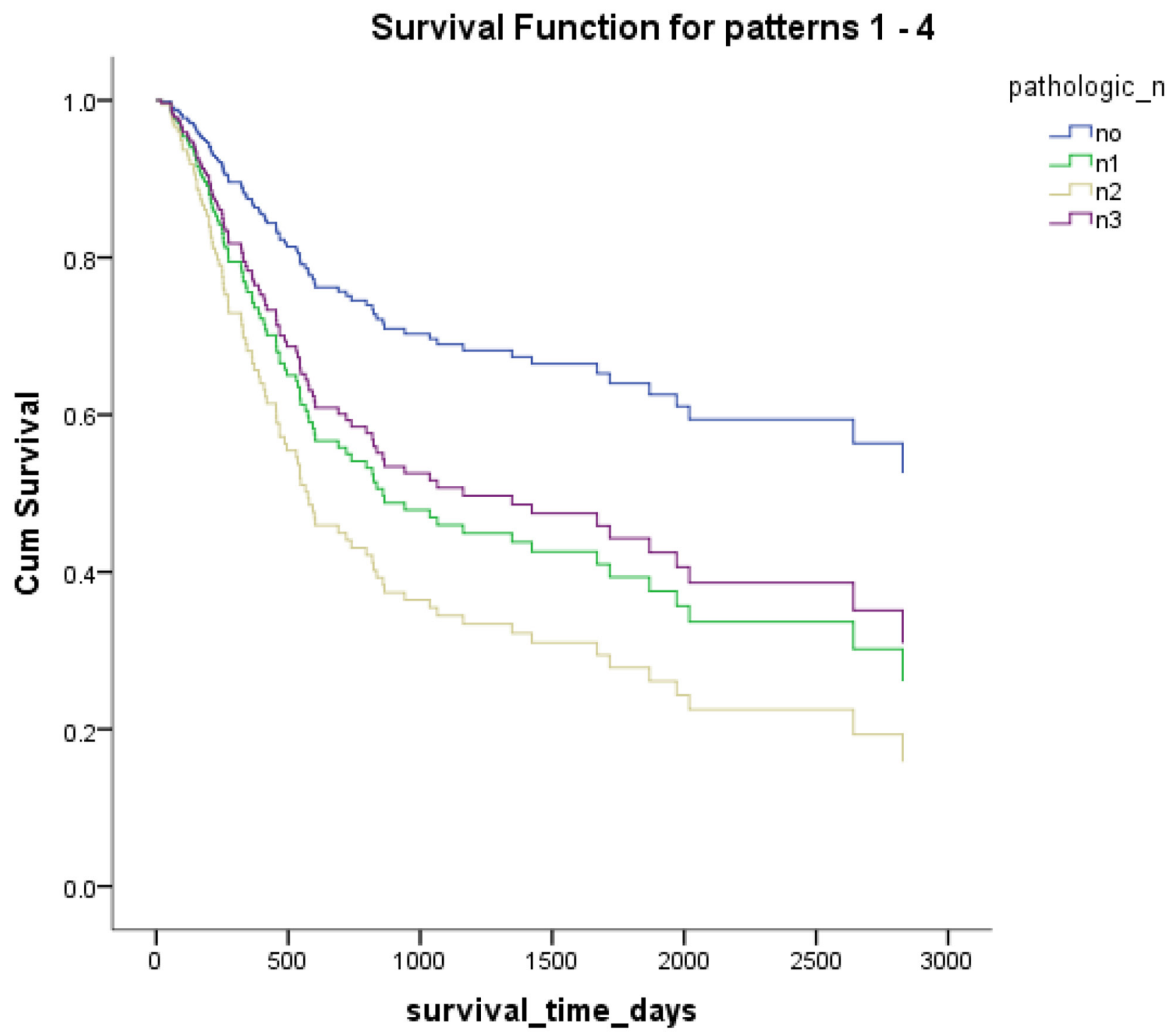

Figure 6: Overall survival according to pathological lymph node metastasis. 
and migration. Furthermore, the overexpression of mex3a was associated with the relapse of Wilms tumors [18]. Baumgart et al. [19] indicated that the expression of mex $3 a$ was similar to the expression of the mitotic marker proliferating cell nuclear antigen. A study [20] demonstrated that the overexpression of $\operatorname{mex} 3 a$ led to the downregulation of CDX2. Gross et al. [21] indicated that CDX2 inhibited cell growth and migration in vitro and the spread of colon tumor cells in vivo, indicating that the reduced expression of CDX2 caused by mex $3 a$ increased the progression of colorectal carcinomas induced by chemical substances.

The results of the independent sample $t$-test revealed significant differences in the expression of mex $3 a$ between cancer tissues and adjacent normal tissues and this result led us to evaluate whether mex $3 a$ was a biomarker of BLCA and whether mex $3 a$ could affect the overall survival of BLCA patients. Hence, the clinical data of 412 patients with BLCA were used to determine the factors associated with mex $3 a$ expression and whether these factors affected OS. However, the analysis of clinical data indicated that OS was not significantly different between the two groups of mex $3 a$ expression. The univariate Cox model showed that the high expression of mex $3 a$ decreased the risk of death by $8.5 \%$ (relative risk $[R R]=0.914$ ) and the multivariate Cox model showed that the high expression of mex $3 a$ decreased the risk of death by $39.4 \% \quad(R R=0.606)$ although the differences were not significant. The decreased risk of death due to increased expression of mex $3 a$ provided discrepancies between the results of the univariate and multivariate Cox models. The result of logistic regression suggests that the cancer subtype strongly affects mex $3 a$ expression. Therefore, the effect of the cancer subtype on the expression of mex3a in the multivariate Cox model might be an important confounding factor. However, previous studies reported conflicting results [12, 19-21].

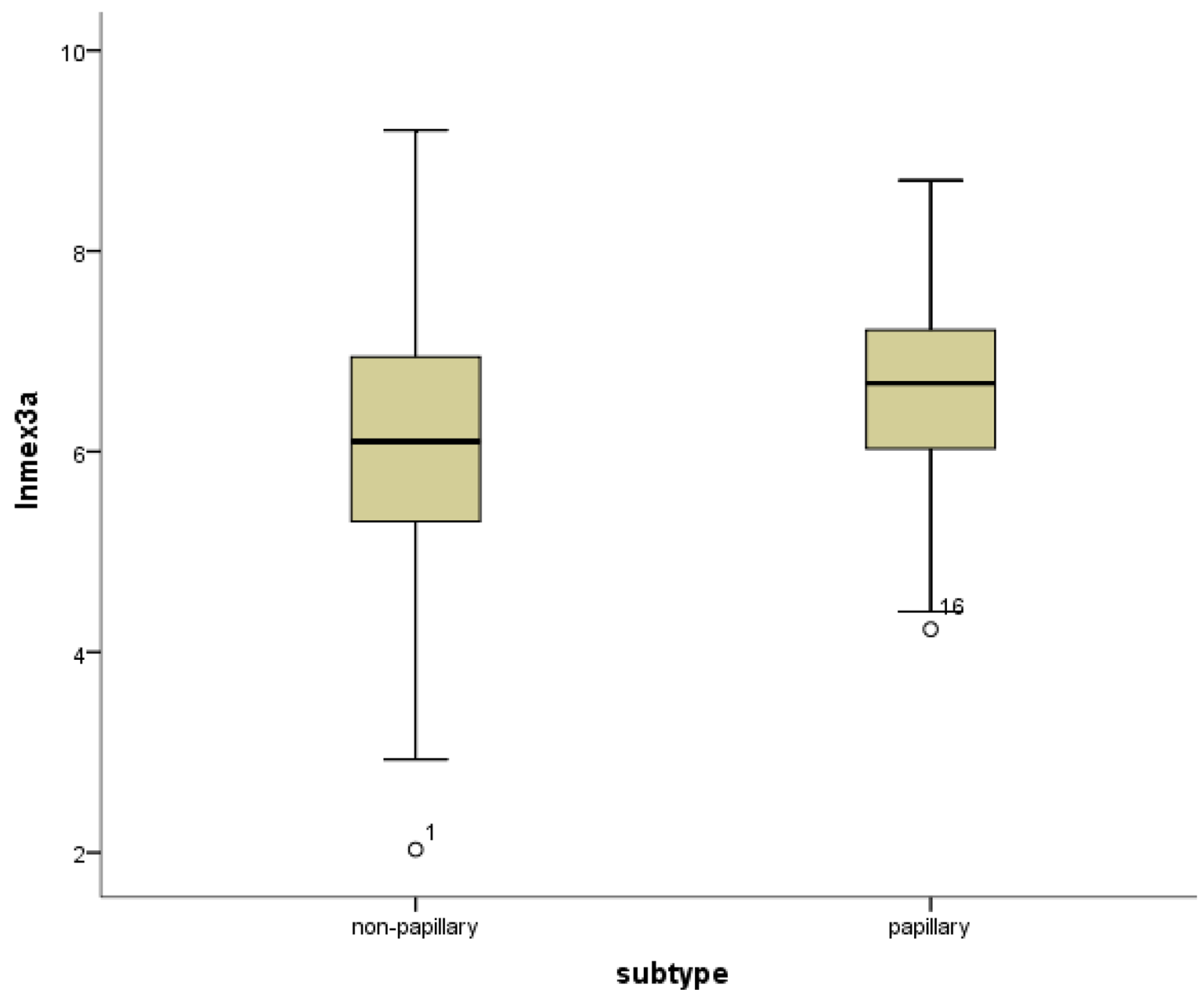

Figure 7: Box-plots shows the $\ln ($ Mex3a) value between the papillary and non-papillary subtypes. 
The univariate Cox model explored different factors separately, and the OS in BLCA was associated with age, cancer status, pathologic stage, pathologic size, pathologic lymph metastasis, and lymphatic vascular invasion. However, among the dummy variables, the absence of significant differences in pathologic lymph metastasis might be because the sample size was not enough for $n 3$ and might lead to significant errors.

The results of the multivariate Cox model adjusted for the mex $3 a$ expression group, and the group that included age, gender, cancer subtype, cancer status, pathologic stage, pathologic size, pathologic lymph metastasis, and lymphatic vascular invasion indicated that only age and cancer status were associated with OS. The cancer status was divided into tumor status and tumor-free status. The former corresponded to the new tumor events after initial treatment whereas the latter corresponded to the status without new tumor after initial treatment. Our results indicated that higher age at diagnosis of BLCA had a 2.653-fold higher risk of death than lower age, and the tumor status had a 6.762-fold higher risk of death than the tumor-free status. The pathologic stage, pathologic size, pathologic lymph metastasis, and pathologic distant lymph metastasis as categorical dependent variables were associated with OS. However, when adjusted for other clinical-pathologic characteristics, these variables might not be associated with OS because of the strong confounding effects of the clinical-pathologic characteristics in the statistical analysis. For example, the pathologic stage was determined by the pathologic size, pathological lymph metastasis, and pathologic distant lymph metastasis according to tumor-node-metastasis (TNM) classification system. Therefore, the interaction between the factors produced a significant effect when pathologic staging and TNM staging were included in the Cox model simultaneously.

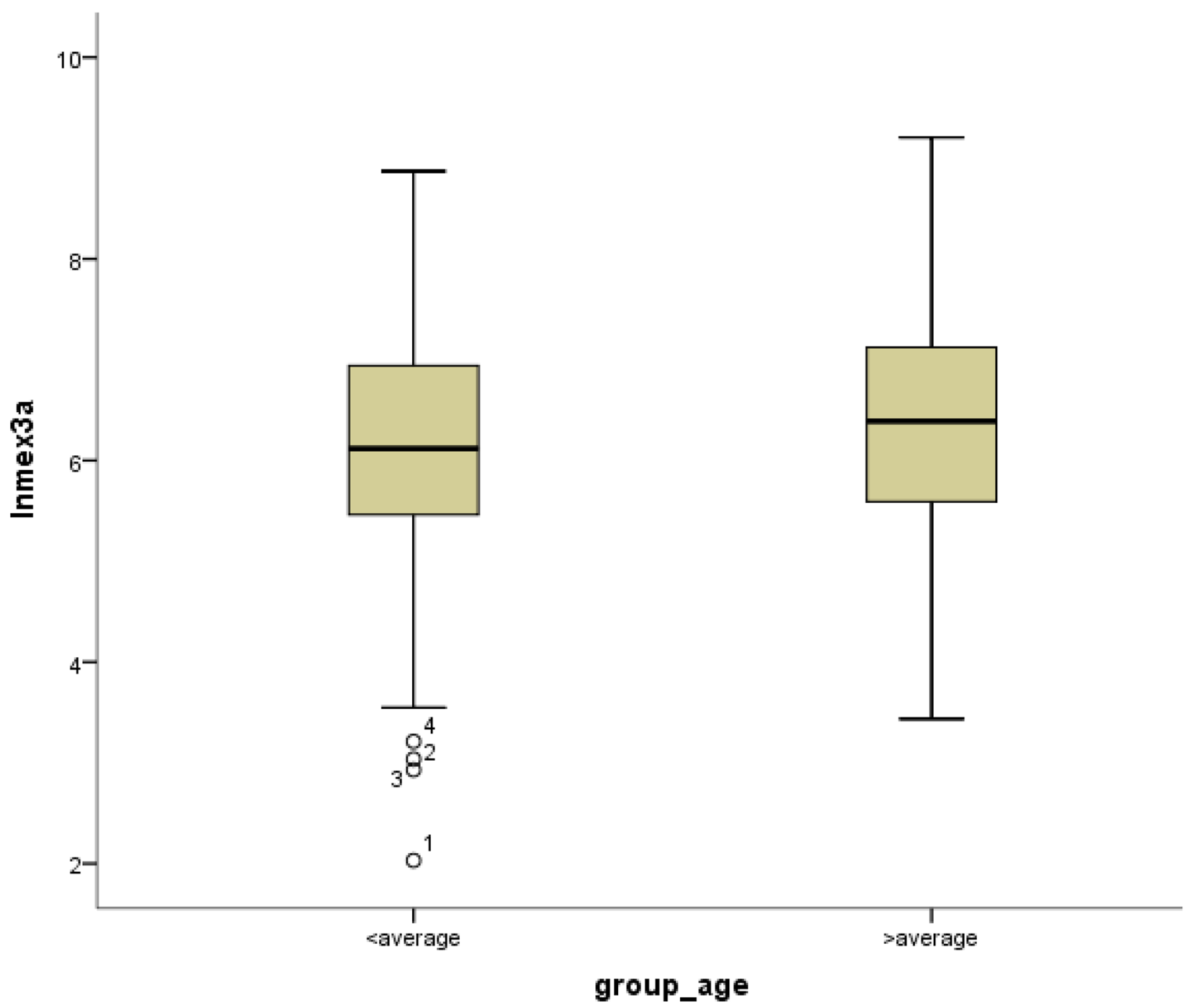

Figure 8: Box-plots shows $\ln (\mathrm{Mex3a})$ value between high and low age. 
BLCA can be divided into invasive tumors and non-invasive tumors. Non-invasive BLCA is divided into papillary and flat types. The lack of infiltration into papillary structures is known as carcinoma in situ, with a high level of cytological characteristics. Papillary tumors include reactive hyperplasia, papilloma, low-grade potential, papillary urothelial proliferation of low malignant potential, and low-grade and high-grade papillary urinary tract epithelial cancers. Flat BLCA has a broad morphology, including reactive hyperplasia, precancerous lesions, and malignant lesions [22]. Most invasive cases of BLCA are confined to the lamina propria [22] and belong to the non-papillary type. In our patients with papillary bladder cancer, the expression of mex $3 a$ was increased, which might indicate that mex $3 a$ could be a classification marker for the BLCA subtypes. Significant differences were found between papillary and non-papillary BLCA tumors, and papillary tumors had a 1.854-fold higher likelihood of presenting a high expression of mex $3 a(P=0.006, \mathrm{OR}=1.854)$. Different factors may affect age; therefore, although the mortality due to bladder cancer increased as age increased [14] and the older group diagnosed with BLCA had a 1.617-fold higher likelihood of presenting increased mex $3 a$ expression $(P=0.027, \mathrm{OR}=1.617)$, no correlation between mex $3 a$ expression and OS was observed.

Previous studies have shown that mex $3 a$ promoted cell division and metastasis and might be a hazard factor in gastric cancer [12] and Wilms tumors [18]. However, in this study, mex $3 a$ did not have a significant effect on the prognosis of BLCA, which might be because of dummy variables in the data from the TCGA database or missing data. However, other unknown factors might have a significant effect on mex $3 a$. Therefore, the effects of mex $3 a$ on OS still need to be elucidated.

In conclusion, the expression level of mex3a was higher in cancer tissues compared with normal adjacent tissues. However, mex3a was not a poor prognostic factor of BLCA. Other factors, including increased age at diagnosis, tumor status, as well as the increase in pathologic stage, pathological size, and pathological lymph metastasis were associated with OS in BLCA. Moreover, mex $3 a$ expression was higher in the papillary type of BLCA than in the non-papillary type.

\section{MATERIALS AND METHODS}

\section{Patients and methods}

The clinical and RNA sequencing (RNA-Seq) expression data for bladder cancer patients were downloaded from the TCGA database (https://tcga-data. nci.nih.gov/tcga/tcgaDownload.jsp). The clinical data were matched with RNA-Seq data, which are used to study the transcriptome profiling and search for novel fusion genes [23]. A total of 412 BLCA samples were available in the TCGA database, and all these samples had corresponding RNA-Seq sequences, with a total of 19 paired samples. Clinical information was acquired from all samples. Clinical information of the BLCA patients was available from 19 paired samples, including age, gender, survival time, and tumor pathologic TNM staging. Mex3a expression was determined in both cancer and normal tissues and the expression levels were normalized.

The expression profile analysis was based on RNASeq data. The original data used for analysis were filtered and standardized before entering the subsequent statistical analysis. We only selected the most expressive transcripts for analysis, and the symbols with fewer than 50 original sequence reads were excluded from the analysis. The trimmed mean of M-values method was used to ensure data normality. This approach used the concept of relative expression between different samples to avoid the misalignment caused by the excessive absolute statistical method.

According to the patient barcode, the patients' clinical information and mex $3 a$ expression level were matched, and RNA-Seq samples with 19 paired samples were also obtained. The mex3a group was classified into two groups (high and low expression) by the median mex3a expression value of 1007.2. The age group was classified into two groups by the median value of the patients' age at first diagnosis of BLCA.

\section{Statistical analysis}

Box-plots were used to express the differences in the mex $3 a$ expression between the groups. The univariate Cox analysis was used to evaluate the OS of BLCA with clinical-pathologic characteristics, respectively, as a categorical dependent variable. The multivariate Cox analysis was used to assess the effect of mex $3 a$ expression on the OS together with other clinical factors (the group that included age, cancer status, cancer subtype, gender, lymphatic vascular invasion, pathological size, pathological metastasis, and pathological stage as categorical dependent variables), and the survivorship curve was expressed to evaluate the OS among different clinical-pathologic characteristics. The univariate logistic regression analysis was used to determine the effects of distinct factors on mex $3 a$ among the dependent variables. An independent sample $t$-test was used to determine the differences in mex $3 a$ expression between tumor tissues and adjacent normal tissues according to data from the 19 paired samples. All data were analyzed with the software SPSS Statistics version 22.0. $P<0.05$ was considered statistically significant.

\section{Author contributions}

Ying Huang designed the research, collected the data and submitted the manuscript; Jing-wen Shi performed the statistical analysis and wrote the manuscript. 


\section{ACKNOWLEDGMENTS}

We are grateful to the staff from the Department of Epidemiology of China Medical University for help in the statistical analysis.

\section{CONFLICTS OF INTEREST}

The authors declare no conflicts of interests.

\section{FUNDING}

This research was supported by the Natural Science Foundation of China (Protocol No. 81371552).

\section{REFERENCES}

1. Siegel RL, Miller KD, Jemal A. Cancer statistics, 2015. CA Cancer J Clin. 2015; 65: 5-29.

2. Chen W, Zheng R, Baade PD, Zhang S, Zeng H, Bray F, Jemal A, Yu XQ, He J. Cancer statistics in China, 2015. CA Cancer J Clin. 2016; 66: 115-32.

3. Zhao XL, Zhao ZH, Xu WC, Hou JQ, Du XY. Increased expression of SPRY4-IT1 predicts poor prognosis and promotes tumor growth and metastasis in bladder cancer. Int J Clin Exp Pathol. 2015; 8: 1954-60.

4. Zhang M, Li W, Hao Z, Zhou J, Zhang L, Liang C. Association Between Twelve Polymorphisms in Five X-ray Repair Crosscomplementing Genes and the Risk of Urological Neoplasms: A Systematic Review and Meta-Analysis. EBioMedicine. 2017; doi:10.1016/j.ebiom.2017.03.009.

5. Lin Y, Sun G, Liu X, Chen Y, Zhang C. Clinical significance of T-cadherin tissue expression in patients with bladder transitional cell carcinoma. Urol Int. 2011; 86: 340-5.

6. Taguchi S, Fukuhara H, Homma Y, Todo T. Current status of clinical trials assessing oncolytic virus therapy for urological cancers. Int J Urol. 2017; doi: 10.1111/iju.13325.

7. Pietzak EJ. The Impact of Blue Light Cystoscopy on the Diagnosis and Treatment of Bladder Cancer. Curr Urol Rep. 2017; $18: 39$.

8. Hassen W, Droller MJ. Current concepts in assessment and treatment of bladder cancer. Curr Opin Urol. 2000; 10: 291-9.

9. Davarpanah NN, Yuno A, Trepel JB, Apolo AB. Immunotherapy: a new treatment paradigm in bladder cancer. Curr Opin Oncol. 2017; doi: 10.1097/ CCO.0000000000000366.

10. Liu J, Li D, Cao L, Wang Z, Li Y, Liu H, Chen G. Elevated preoperative plasma fibrinogen level is an independent predictor of malignancy and advanced stage disease in patients with bladder urothelial tumors. Int J Surg. 2016; 36: 249-54.

11. Wang CT, Li CF, Wu WJ, Huang CN, Li CC, Li WM, Chan TC, Liang PI, Hsing CH, Liao KM. High Expression of
17 $\beta$-hydroxysteroid Dehydrogenase Type 2 is Associated with a Better Prognosis in Urothelial Carcinoma of the Urinary Tract. J Cancer. 2016; 7: 2221-30.

12. Jiang H, Zhang X, Luo J, Dong C, Xue J, Wei W, Chen J, Zhou J, Gao Y, Yang C. Knockdown of hMex-3A by small RNA interference suppresses cell proliferation and migration in human gastric cancer cells. Mol Med Rep. 2012; 6: 575-80.

13. Sun X, Hoadley KA, Kim WY, Furberg H, Olshan AF, Troester MA. Age at diagnosis, obesity, smoking, and molecular subtypes in muscle-invasive bladder cancer. Cancer Causes Control. 2017; doi:10.1007/ s10552-017-0885-z.

14. Han SJ, Zhang SW, Chen WG, Li CL. Analysis of the status quo and trends: mortality in patients with bladder cancer in China. Journal of Modern Urology. 2013; 18: 228-32.

15. Grishin NV. KH domain: one motif, two folds. Nucleic Acids Res. 2001; 29: 638-43.

16. Draper BW, Mello CC, Bowerman B, Hardin J, Priess JR. MEX-3 is a $\mathrm{KH}$ domain protein that regulates blastomere identity in early C. elegans embryos. Cell. 1996; 87: 205-16.

17. Buchet-Poyau K, Courchet J, Le HH, Séraphin B, Scoazec JY, Duret L, Domon-Dell C, Freund JN, Billaud $\mathrm{M}$. Identification and characterization of human Mex-3 proteins, a novel family of evolutionarily conserved RNAbinding proteins differentially localized to processing bodies. Nucleic Acids Res. 2007; 35: 1289-300.

18. Krepischi AC, Maschietto M, Ferreira EN, Silva AG, Costa SS, da Cuinha IW, Barros BD, Grundy PE, Rosenberg C, Carraro DM. Genomic imbalances pinpoint potential oncogenes and tumor suppressors in Wilms tumors. Mol Cytogenet. 2016; 9: 20.

19. Baumgart M, Groth M, Priebe S, Savino A, Testa G, Dix A, Ripa R, Spallotta F, Gaetano C, Ori M, Terzibasi TE, Guthke R, Platzer M, et al. RNA-seq of the aging brain in the short-lived fish N. furzeri - conserved pathways and novel genes associated with neurogenesis. Aging Cell. 2014; 13: 965-74.

20. Pereira B, Sousa S, Barros R, Carreto L, Oliveira P, Oliveira C, Chartier NT, Plateroti M, Rouault JP, Freund JN, Billaud M, Almeida R. CDX2 regulation by the RNA-binding protein MEX3A: impact on intestinal differentiation and stemness. Nucleic Acids Res. 2013; 41: 3986-99.

21. Gross I, Duluc I, Benameur T, Calon A, Martin E, Brabletz T, Kedinger M, Domon-Dell C, Freund JN. The intestinespecific homeobox gene $\mathrm{Cdx} 2$ decreases mobility and antagonizes dissemination of colon cancer cells. Oncogene. 2008; 27: 107-15.

22. Wei JG, Teng XD. 2016 WHO urinary system and male genital tumor classification (bladder) interpretation. Journal of Practical Oncology. 2016;31: 331-5.

23. Wang Z, Gerstein M, Snyder M. RNA-Seq: a revolutionary tool for transcriptomics. Nat Rev Genet. 2009; 10: 57-63. 